BULL. AUSTRAL. MATH. SOC.

VOL. 8 (1973), $211-214$.

\title{
A constructive proof of
}

\section{Gelbaum's theorem on tensor products}

\section{David A. Robbins}

\begin{abstract}
A constructive proof is given of Gelbaum's result that the maximal ideal space of the tensor product of commutative Banach algebras is homeomorphic to the cartesian product of the maximal ideal spaces.
\end{abstract}

It is a well-known result of Gelbaum [1] that if $A$ and $B$ are commutative Banach algebras and $\Delta(A)$ and $\Delta(B)$ their respective maximal ideal spaces, then the maximal ideal space $\Delta\left(A \otimes_{\gamma} B\right)$ of $A \otimes_{\gamma} B$ is naturally homeomorphic to $\Delta(A) \times \Delta(B)$, where $\gamma$ is the greatest crossnorm. We are concerned with giving a much shorter, constructive proof of this result, using only the universal mapping property of tensor products for the cross-norm $\gamma$, and the known representations of elements of $A \otimes_{\gamma} B$.

Recall that any element $z \in A \otimes_{\gamma} B$ may be written $z=\sum_{i=1}^{\infty} a_{i} \otimes b_{i}$, where $\sum_{i=1}\left\|a_{i}\right\|\left\|b_{i}\right\|<\infty$, and that, if $S$ and $T$ are continuous endomorphisms of $A$ and $B$, respectively, then

$$
\|(S \otimes T)(z)\|=\left\|\sum_{i=1}^{\infty}\left(S a_{i}\right) \otimes\left(T b_{i}\right)\right\| \leq\|S\|\|T\|\|z\| .
$$

THEOREM (Gelbaum). Let $A$ and $B$ be commutative Banach algebras. Then $\Delta\left(A \otimes_{\gamma} B\right)$ is weak-* homeomorphic to $\Delta(A) \times \Delta(B)$.

Receŕved 2 November 1972. 
Proof. Let $\varphi: \Delta(A) \times \Delta(B) \rightarrow\left(A \otimes_{\gamma} B\right) *$ be the map such that $\varphi(f, g)=f \otimes g$. It is easily checked that $f \otimes g \in \Delta\left(A \otimes_{\gamma} B\right)$ and that $\varphi$ is injective.

We claim $\varphi$ is surjective: let $h \in \Delta\left(A \otimes_{\gamma} B\right)$, and let $z \in A \otimes_{\curlyvee} B$, written $z=\sum_{i=1}^{\infty} a_{i} \otimes b_{i}$, be an identity mod $h$. Define $f_{z} \in A^{*}$ by $f_{z}(a)=h\left(\sum_{i} a a_{i} \otimes b_{i}\right)$, for $a \in A$. (We note that $\sum_{i} a a_{i} \otimes b_{i}$ is well-defined, by the universal mapping property. $\}$ It is clear that $f_{z}$ is Iinear on $A$, and, if $a, a^{\prime} \in A$, we have

$$
\begin{aligned}
f_{z}\left(a a^{\prime}\right) & =h\left(\sum_{i} a a^{\prime} a_{i} \otimes b_{i}\right)=h\left(\left(\sum_{i} a a^{\prime} a_{i} \otimes b_{i}\right)\left(\sum_{i} a_{i} \otimes b_{i}\right)\right) \\
& =h\left(\sum_{i, j} a a^{\prime} a_{i} a_{j} \otimes b_{i} b_{j}\right) \\
& =h\left(\left(\sum_{i} a a_{i} \otimes b_{i}\right)\left(\sum_{i} a^{\prime} a_{i} \otimes b_{i}\right)\right) \\
& =f_{z}(a) f_{z}\left(a^{\prime}\right),
\end{aligned}
$$

so that $f_{z}$ is multiplicative on $A$, and hence $f_{z} \in \Delta(A)$. Finally, $f_{z}$ is independent of our choice of identity $\bmod h$. For, if $z^{\prime}=\sum_{i} a_{i}^{\prime} \otimes b_{i}^{\prime}$ is another identity $\bmod h$, and if $a \in A$, we have

$$
\begin{aligned}
f_{z}(a) & =h\left(\sum_{i} a a_{i} \otimes b_{i}\right)=h\left(\left(\sum_{i} a a_{i} \otimes b_{i}\right)\left(\sum_{i} a_{i}^{\prime} \otimes b_{i}^{\prime}\right)\right) \\
& =h\left(\sum_{i, j} a a_{i} a_{j}^{\prime} \otimes b_{i} b_{j}^{\prime}\right) \\
& =h\left(\left(\sum_{i} a a_{i}^{\prime} \otimes b_{i}^{\prime}\right)\left(\left[\sum_{i} a_{i} \otimes b_{i}\right)\right)=f_{z^{\prime}}(a) .\right.
\end{aligned}
$$

We now define $g_{z} \in \Delta(B)$ in a similar manner, and claim $h=f_{z} \otimes g_{z}$. Let $x \in A \otimes_{\gamma} B$ be of form $x=a \otimes b$. Then 


$$
\begin{aligned}
\left(f_{z} \otimes g_{z}\right)(a \otimes b) & =f_{z}(a) g_{z}(b)=h\left(\sum_{i} a a_{i} \otimes b_{i}\right) h\left(\sum_{i} a_{i} \otimes b b_{i}\right) \\
& =h\left(\sum_{i, j} a a_{i} a_{j} \otimes b b_{i} b_{j}\right) \\
& =h\left((a \otimes b)\left(\sum_{i} a_{i} \otimes b_{i}\right)\left(\sum_{i} a_{i} \otimes b_{i}\right)\right) \\
& =h(a \otimes b) .
\end{aligned}
$$

Since elements of form $a \otimes b$ span a dense subspace of $A \otimes_{\gamma} B$, we see that $h=f_{z} \otimes g_{z}$, and hence $\varphi$ is surjective.

If $h \in \Delta\left(A \otimes_{\gamma} B\right)$, we now write $h=f \otimes g$, where $f \in \Delta(A)$ and $g \in \Delta(B)$.

It is easily shown that $\varphi$ is continuous. Suppose that $\left(f_{p}, g_{p}\right) \stackrel{p}{\longrightarrow}(f, g)$ in $\Delta(A) \times \Delta(B)$. If $z \in A \otimes_{\gamma} B$ is of form $z=\sum_{i=1}^{n} a_{i} \otimes b_{i} \quad$ (that is, if $z \in A \otimes B$ ), it is clear that $\left(f_{p} \otimes g_{p}\right)(z) \stackrel{P}{\longrightarrow}(f \otimes g)(z)$. Now, let $z \in A \otimes_{\gamma} B$, and let $\varepsilon>0$ be given. Choose $z^{\prime} \in A \otimes B$ such that $\left\|z-z^{\prime}\right\|<\varepsilon / 3$. Then, if $p$ is sufficiently large, we have

$$
\begin{aligned}
\mid\left(f_{p} \otimes g_{p}\right)(z) & -(f \otimes g)(z)|\leq|\left(f_{p} \otimes g_{p}\right)(z)-\left(f_{p} \otimes g_{p}\right)\left(z^{\prime}\right) \mid \\
& +\left|\left(f_{p} \otimes g_{p}\right)\left(z^{\prime}\right)-(f \otimes g)\left(z^{\prime}\right)\right|+\left|(f \otimes g)\left(z^{\prime}\right)-(f \otimes g)(z)\right|<\varepsilon,
\end{aligned}
$$

since $\left\|f_{p} \otimes g_{p}\right\|=\left\|f_{p}\right\|\left\|g_{p}\right\| \leq 1$ and $\|f \otimes g\|=\|f\|\|g\| \leq 1$, so that $\varphi$ is continuous.

Finally, we claim $\varphi^{-1}$ is continuous. Let $h_{p}=f_{p} \otimes g_{p} \stackrel{p}{\longrightarrow} h=f \otimes g$ be a convergent net in $\Delta\left(A \otimes_{\gamma} B\right)$. We claim $f_{p} \stackrel{p}{\longrightarrow} f$ and $g_{p} \stackrel{p}{\longrightarrow} g$ in $\Delta(A)$ and $\Delta(B)$, respectively. To show $f_{p} \stackrel{p}{\longrightarrow} f$, it will suffice to show that every subnet $\left\{f_{q}\right\}$ of $\left\{f_{p}\right\}$ has a subsubnet $\left\{f_{s}\right\}$ such that $f_{\varepsilon} \stackrel{s}{\longrightarrow} f$. Let $\left\{f_{q}\right\}$ be a subnet of $\left\{f_{p}\right\}$. Since $\overline{\Delta(A)}$ is compact, there is a subnet $\left\{f_{r}\right\}$ of $\left\{f_{q}\right\}$ which converges 
to some $f^{\prime}$ in $\overline{\Delta(A)}$. By similar reasoning, there exists a subnet $\left\{g_{s}\right\}$ of $\left\{g_{p}\right\}$ such that $g_{\boldsymbol{g}} \stackrel{\boldsymbol{\varepsilon}}{\longrightarrow} g^{\prime}$ for some $g^{\prime}$ in $\overline{\Delta(B)}$. We then have $f_{s} \otimes g_{s} \stackrel{\varepsilon}{\longrightarrow} f^{\prime} \otimes g^{\prime}$, and, since $\left\{f_{s} \otimes g_{s}\right\}$ is a subnet of $\left\{f_{p} \otimes g_{p}\right\}$, we have $f_{\boldsymbol{g}} \otimes g_{\boldsymbol{s}} \stackrel{\boldsymbol{s}}{\longrightarrow} f \otimes g$. Thus, $f^{\prime} \otimes g^{\prime}=f \otimes g$, so that $f^{\prime} \in \Delta(A)$ and $g^{\prime} \in \Delta(B)$. Then, since $\varphi$ is ingective, $f^{\prime}=f$. Similarly, we may show that $g_{p} \stackrel{p}{\longrightarrow} g$, so that $\varphi^{-1}$ is continuous, and the homeomorphism is established. //

With suitable modifications, our proof will serve to show the same result for any tensor product $A \otimes_{\alpha} B$ of commutative Banach algebras $A$ and $B$, where $\lambda \leq \alpha \leq \gamma$ is any uniform cross-norm such that $A \otimes_{\alpha} B$ is a Banach algebra and where $\lambda$ is the "least" cross-norm.

\section{Reference}

[1] Bernard R. Gelbaum, "Tensor products of Banach algebras", Canad. J. Math. 11 (1959), 297-310.

Department of Mathematics,

Trinity College,

Hartford,

Connecticut,

USA. 